\title{
Resiliently evolving supply-demand networks
}

\author{
Nicolás Rubido, ${ }^{1,2, *}$ Celso Grebogi, ${ }^{1}$ and Murilo S. Baptista ${ }^{1}$ \\ ${ }^{1}$ Institute for Complex Systems and Mathematical Biology, University of Aberdeen, King's College, AB24 3UE Aberdeen, United Kingdom \\ ${ }^{2}$ Instituto de Física, Facultad de Ciencias, Universidad de la República, Iguá 4225, Montevideo 11200, Uruguay
}

(Received 27 June 2013; revised manuscript received 2 October 2013; published 9 January 2014)

\begin{abstract}
The ability to design a transport network such that commodities are brought from suppliers to consumers in a steady, optimal, and stable way is of great importance for distribution systems nowadays. In this work, by using the circuit laws of Kirchhoff and Ohm, we provide the exact capacities of the edges that an optimal supply-demand network should have to operate stably under perturbations, i.e., without overloading. The perturbations we consider are the evolution of the connecting topology, the decentralization of hub sources or sinks, and the intermittence of supplier and consumer characteristics. We analyze these conditions and the impact of our results, both on the current United Kingdom power-grid structure and on numerically generated evolving archetypal network topologies.
\end{abstract}

DOI: 10.1103/PhysRevE.89.012801

PACS number(s): 89.75.Fb, 41.20.-q, 89.40.-a, 89.75.Hc

Networks are ubiquitous in nature and in manmade systems. Power and gas networks bring light and heat to our homes, telecommunication networks allow us to be entertained and to browse for information, and distribution networks allow manufacturers to supply foodstock and other products to the demand chain. In all of these cases, a basic problem needs to be addressed: how to create a network that transports the maximum load (messages, power, vehicles, consumer products, etc.) that can be moved from one point in the network to another by following optimal paths (for instance, making the energy consumption minimal), without surpassing any edge or node capacity (the maximum load that an edge or node can handle), and generates a steady stable flow. Such problem is dealt with in this work.

We understand that a supply-demand network is stable when the system is not vulnerable to modifications in the network's connectivity, a switch from hub suppliers to decentralized smaller producers, or changes in the location of suppliers and/or consumers that may cause overload failures to occur. The network is vulnerable when any edge load exceeds its edge capacity and, thus, a failure occurs. Such a distribution network has a steady transport when flows (the time ratio that commodities are transferred between nodes) are constant. It is optimal when the transport is done such that the cost is minimum.

Our problem is, in part, that which flow network theory [14], a theory that roots back to Kirchhoff [1], tries to decipher, i.e., answering what are the current flows in each edge of an electrical circuit as a set of voltages are applied to some nodes. The solution is then achieved by solving Kirchhoff's equations. It is related to the probability that a random walker starts at the source and finishes at the sink [5] and to first-passage times at each node [6]. In order to model a supply-demand network, we solve the inverse problem. We

\footnotetext{
*n.rubido.obrer@abdn.ac.uk
}

Published by the American Physical Society under the terms of the Creative Commons Attribution 3.0 License. Further distribution of this work must maintain attribution to the author(s) and the published article's title, journal citation, and DOI. calculate voltages (loads) when input-output currents (flows) are given, using a conservative, steady, minimal cost transport system: Kirchhoff's flow network. This means loads are carried optimally from the source (supplier) to the sink (consumer) without losses. It is related to finding the shortest paths and community structures on weighted networks $[7,8]$.

In particular, Kirchhoff's flow network model is used not only to express electrical flow in circuits [9], but also to establish systems ecology relationships [10], biologically inspired steady-state transport systems [11], and fractures in materials $[12,13]$. Although the relationship between flows and loads in these models is restricted to be linear and conservative, the complexity in the mathematical treatment of the equations due to the topology structure is still demanding. Thus, most flow network solutions are based on optimization schemes [3], and the results are complex and not easy to relate to other relevant parameters of the problem, such as node degrees. Moreover, if the network evolves in time (the connecting topology changes with nodes and/or edges appearing and/or disappearing), then predictions, controlling cascade of failures, and analytical solutions are scarce [14-17].

In this work, we provide analytical expressions for the edge capacities that a steady optimal supply-demand network should have to operate stably under perturbations by using Kirchhoff's flow network model. The perturbations we consider constitute some possible evolution factors that supplydemand networks are subjected to, such as the switching from hub suppliers to multiple smaller producers, intermittent supplying and consuming nodes, and node and edge additions or removals.

We apply our edge capacity analytical results both to the current United Kingdom (U.K.) power grid and to numerically generated evolving archetypal network topologies. We discuss the design of a modern steady-state stable power-grid system and we find that most topology modifications have a powerlaw behavior of the analytically derived edge capacities as nodes or edges are added (or removed, which in our model constitutes the inverse process of addition) to the network. Our results and conclusions are general and applicable to any other system that is modeled by the Kirchhoff flow network model in its steady state. Furthermore, they are related to standard network characteristics and allow the prediction of failures 
due to overloads by observing the load at any given edge and comparing it to our edge capacity values [Eq. (3)].

To achieve an analytical solution to our problem, we initially assume that the network structure, the location of the supplier(s) and consumer(s), and the total amount of commodities produced and consumed are known, but loads and flows in every edge need to be calculated. We let loads be linearly related to the flows by

$$
l_{i j}^{(s t)}=R_{i j} f_{i j}^{(s t)} \text {. }
$$

The left-hand side of Eq. (1) is the load being transferred across the edge connecting nodes $i$ and $j$ of the network given a source located at node $s$ and a sink located at node $t$. The extension to many sources and sinks adds more upper indexes to the equation (see Supplemental Material [18]). In our model, Eq. (1) is Ohm's law, and $l_{i j}^{(s t)}=V_{i}^{(s t)}-V_{j}^{(s t)}$ represents the voltage difference between nodes $i$ and $j$ in an electric circuit, where a given current enters the circuit at node $s$ and leaves at node $t$ [9]. However, it can also represent the altitude difference in a river channel network where the highest place is located at point $s$ and the lowest place is located at point $t$. The right-hand side of Eq. (1) is composed of the resistance $R_{i j}$ of the edge to transport and the unknown flow $f_{i j}^{(s t)}$, which the edge has for that particular location of the supply-demand nodes. In our model, it is the edge's electrical resistance $R_{i j}$ times the electrical current $f_{i j}^{(s t)}=I_{i j}^{(s t)}$. For the river channel, it could be the channel resistance of a Poiseuille flow times the mass flow velocity transported by the $i j$ th river channel. For example, in systems ecology, Eq. (1) relates the storage quantities $Q$ with the outflows $J$ via time constants $T$ [10].

Because we assume the model to be conservative, the net flow at any node $i$ in the network is null $\left(\sum_{j=1}^{N} f_{i j}^{(s t)}=0\right)$, with the exception of the source (whose net flow is represented by $I$ ) and the sink (whose net flow is represented by $-I$ ) nodes. This guarantees that flows are carried optimally from the source(s) to the $\operatorname{sink}(\mathrm{s})$. Then, the net flow at node $i$ is modeled by Kirchhoff's first law,

$$
\sum_{j=1}^{N} f_{i j}^{(s t)}=I\left(\delta_{i s}-\delta_{i t}\right)=\sum_{j=1}^{N} W_{i j} l_{i j}^{(s t)},
$$

where $\delta_{i s}$ is the Kronecker delta function $\left(\delta_{i s}=1\right.$ if $\left.i=s\right)$ and $W_{i j}=1 / R_{i j}$ is the matrix representation of the network structure, where $R_{i j}=R_{j i}$. In particular, $R_{i j}=\infty$ if nodes $i$ and $j$ are not directly connected, and in an unweighted network, $R_{i j}=1$ for all the connected nodes. Thus, $W_{i j}$ is a weighted adjacency matrix with entries that are either $>0$ (nodes $i$ and $j$ are adjacent, i.e., $A_{i j}=1$ ) or 0 ( $i$ and $j$ are disjoint, i.e., $A_{i j}=0$ ).

We also make use of the equivalent resistance $\rho_{i j}(\mathbf{W})$, which is a network structure characteristic [9,19-21]. It is found analytically from the weighted Laplacian matrix $\mathbf{G}$ eigenvalues and eigenvectors $\left(\mathbf{G}=\mathbf{D}-\mathbf{W}\right.$, with $D_{i j}=\delta_{i j} k_{j}$, and $k_{i}=\sum_{i=1}^{N} W_{i j}$, with $N$ being the number of nodes in the network). This quantity allows one to express any connected arbitrary topology (defined with or without weighted edges) to an effective weighted complete network. In other words, the complexity of the topology is transformed into a complex distribution of weights of an all-to-all complete graph.
Our first analytical result is the exact derivation of the edge capacity $C_{i j}^{(s t)}$ that a supply-demand network must have in order to operate stably and avoid overloads, namely, $\left|l_{i j}^{(s t)}\right| \leqslant C_{i j}^{(s t)}$. We obtain that $C_{i j}^{(s t)}$ is a function of the equivalent resistance $\rho_{i j}$ of the edge and is proportional to the total amount of commodities per unit of time that are produced by the suppliers, namely, the total input $I$. Consequently, the value of $C_{i j}^{(s t)}$ is independent of where the suppliers and consumers are located within the network $\left(C_{i j}^{(s t)}=C_{i j}\right)$. Specifically, we derive that the exact value for the edge capacity is

$$
C_{i j} \equiv I \rho_{i j},
$$

where the functional $\rho_{i j}(\mathbf{W})$ is the equivalent resistance between nodes $i$ and $j$ [9].

The edge capacity in Eq. (3) is the maximum load that each existing edge of the supply-demand network must be able to handle to secure a steady-state stable distribution, regardless of the location of the producer and consumer and regardless of whether, instead of a single supplier and consumer, there are many with arbitrary spatial distributions [the demonstration of Eq. (3) from Eq. (2) is given in the Supplemental Material [18]]. To derive the exact values for node capacity $C_{i}$, we perform a summation over all edge capacities $C_{i}=\sum_{j=1}^{N} C_{i j}$, which is feasible because our model of flow network is conservative. Hence, the node and edge capacities are not independent quantities.

A cascade of failures on networks is often studied by analyzing how attacks and/or overloads occur when a load surpasses the node capacity. Such a node capacity is conjectured to have, with some tuning parameters, a linear relationship with the initial load distribution [16,17]. This assumption allows one to draw conclusions on how the network structure should be designed to avoid failures due to overloads. Here, we show that the capacity-load relationship is given by $\rho_{i j}[E q$. (3)], and it is derived from finding the edge's maximum loads for any of the discussed network evolution processes. In the cases where the physical edge capacity is preassigned, such as in a fuse network (a model that explains fractures in materials [12,13]), Eq. (3) predicts exactly which edges will overload due to the perturbations. This can still aid in the prevention of a cascade of failures, as it detects the vulnerable edges exactly.

The second analytical result we find is that all $C_{i j}$ defined by $E q$. (3) are bounded by the inverse of the largest $\left[\lambda_{N-1}(\mathbf{G})\right]$ and the smallest nonzero $\left[\lambda_{1}(\mathbf{G})\right.$, also known as spectral gap] weighted Laplacian matrix $G_{i j}$ eigenvalues [22,23]. In particular, we find that

$$
\frac{2 I}{\lambda_{N-1}}\left(1-\delta_{i j}\right) \leqslant C_{i j} \leqslant \frac{2 I}{\lambda_{1}}\left(1-\delta_{i j}\right) .
$$

Moreover, these eigenvalues are related to the minimal and maximal degrees of the network. Equation (4) provides a way to modify the network topology (adding or removing nodes and edges), keeping the capacity values bounded by considering simple rules from minimal information about the topology [the derivation of Eq. (4) and the relationship of these bounds to the node degrees is provided in the Supplemental Material [18].

As a practical proof of concept and a way to illustrate these analytical results, we apply them both to the 
U.K. power-grid structure [24] and numerically generated random [25] and small-world [26] topologies. In addition, we discuss the following perturbations: (i) power generator decentralization (changing from centralized high-power generators to distributed smaller generators), (ii) source-sink intermittency (the inclusion of suppliers and consumers, such as renewable sources, storage systems, and electric cars, all possibly changing locations within the network), and (iii) connectivity modifications. These are some of the most important perturbations that modern power-grid systems are having to deal with. We address cases (i) and (ii) using the real U.K. power-grid structure. For case (iii), we focus the analysis on how the connectivity modifications affect the edge's capacity by deriving them for numerically generated networks.

Since the capacity is linearly related to $\rho_{i j}$ [Eq. (3)], to obtain the influence of the topology on the loads we first calculate the U.K. power grid $\rho_{i j}$ by assuming $R_{i j}=1$ for all connected nodes, i.e., $W_{i j}=A_{i j}$. The resultant set of $\rho_{i j}$ is represented by $\rho(A)$ in Fig. 1 . We then calculate $\rho_{i j}$ considering the edge's resistance in MVA units (mega Volt-Ampere) [24], i.e., $W_{i j}=1 / R_{i j}$. This set is represented by $\rho(W)$ in Fig. 1 . The bounds in terms of the maximum and minimum eigenvalues [Eq. (4)] are shown by the vertical dashed lines in each case.

From an engineering point of view, the steady-state stability of a power network is the capability of the system to maintain the power transmitted between any two nodes below the edge capacity (namely, the maximal load that the connecting power line can handle) when perturbations are applied to the network. Figure 1 shows the U.K.'s power-grid equivalent resistance distribution, i.e., our theoretical edge capacity distribution, from taking into account only its connecting topology $(A)$ or its resistive properties $(W)$. If we neglect the reactance and inductance characteristics of the real power lines and we model the power grid by a conservative linear flow network model such as Eq. (1), then assigning edge capacities to the edges in the power grid drawn from the $\rho$ distribution guarantees the steady-state stability of the system. Such a power-grid system

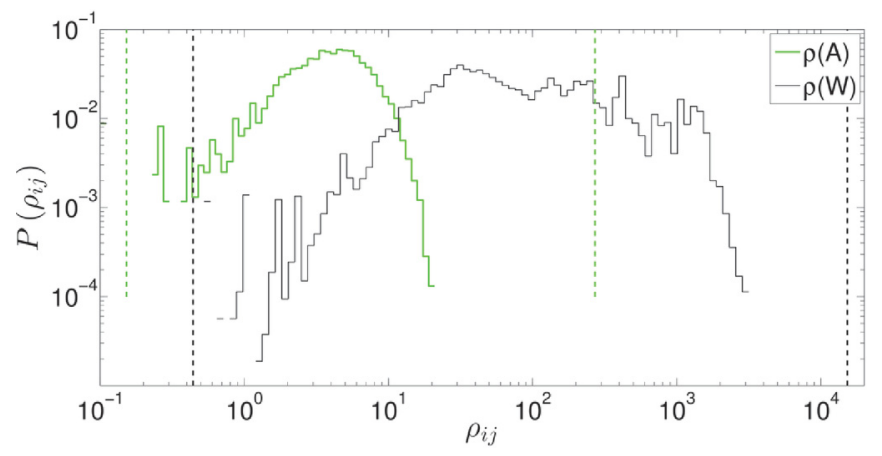

FIG. 1. (Color online) The probability density functions of the equivalent resistance $\rho_{i j}$ (stairlike lines) for the U.K. power-grid adjacency (green) and resistance structure (black). The respective bounds [Eq. (4)] are shown by vertical dashed lines. The units of $\rho_{i j}$ are in MVA (as the power-line resistances found from [24]) and include all three of the U.K.'s major transmission companies (SHETL, SPT, and NGET). is resilient to changing from hub generators to distributed sources [case (i)] or having intermittent sources and sinks [case (ii)].

In case (i), a single source is substituted by multiple sources while maintaining the same inflow of power and keeping the same network connectivity. Specifically, the transformation that leads from a single source-sink problem to one of many sources and sinks does not change the topology. No node or edge is added to the topology to account for new sources or sinks. The transformation identifies nodes that were junctions (where the net flow was conserved, i.e., the inflow is equal to the outflow) in the original single source-sink problem as new sources or sinks in the many source-sink problem. In summary, $I$ is kept constant in the transformation, some nodes of the network are now identified as sources and sinks which were previously junctions, and the connectivity between nodes does not change. In that case, our first analytical result for $C_{i j}$ [Eq. (3)] predicts that the new maximum loads are always less than the capacity value, $\left|l_{i j}^{\left(s_{1}, s_{2}, \ldots, t_{1}, t_{2}, \ldots\right)}\right|<\left|l_{i j}^{(s t)}\right| \leqslant I \rho_{i j}$. In case (ii), given the intermittency property of renewable sources and electric car power stations, the supply-demand network behaves as if sources and sinks keep changing locations with time. In other words, the system explores various configurations of the many source-sink problems for a fixed topology and total input $I$. This situation is also contemplated in our Eq. (3). For a single-source single-single network, the given capacity value for edges is robust and remains unchanged for any source-sink configuration, predicting that overloads will be avoided.

On the contrary, the modifications to the connecting topology [case (iii)] change the value of the edge capacities. This is a consequence of the change in the structure of the network, which, in turn, changes the value of $\rho_{i j}$ in every edge and redistributes the flows. In this case, in order to draw conclusions about edge capacities for a power grid such as the U.K., one needs a dynamic picture of the network topology as it evolves.

We find that to design edge capacities with fixed margins as the supply-demand network topology changes, the minimum and maximum eigenvalues of the weighted Laplacian matrix [Eq. (4)] must be kept fixed or, at least, the minimal and maximal node degrees must be fixed (see Supplemental Material [18]). In this sense, the changes in the topology are contained within the bounds of the initial edge capacities. For the U.K. power grid, this corresponds to modifications in the topology or resistance such that the vertical dashed lines in Fig. 1 remain fixed. Resilience is then enforced by using the upper bound of Eq. (4) for every edge. This approach requires the use of equal capacity lines for the entire network, namely, $2 I / \lambda_{1}$.

In general, we find that if a supply-demand network needs to be designed with similar edge capacities, the topology has to be set such that it resembles as much as possible a complete graph (which has all its non-null eigenvalues equal to the node degrees). This narrows the values that $\rho_{i j}$ can take. On the other hand, if the range of $C_{i j}$ values sought needs to be as broad as possible, the network should be designed to include nodes with high node degree (this increases the largest eigenvalue, thus diminishing the lower bound for the edge capacity) and well-defined communities or nodes 
(a)

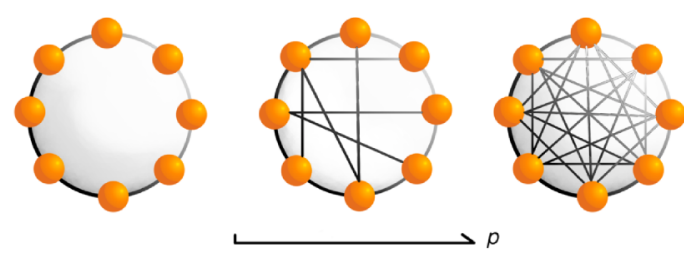

(b)
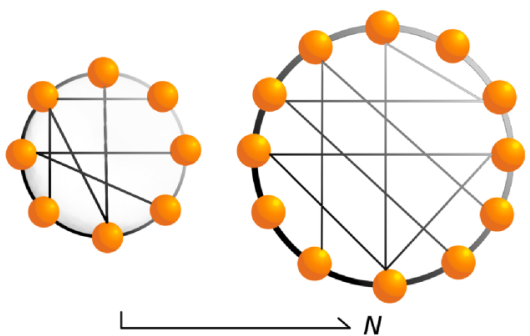

(c)
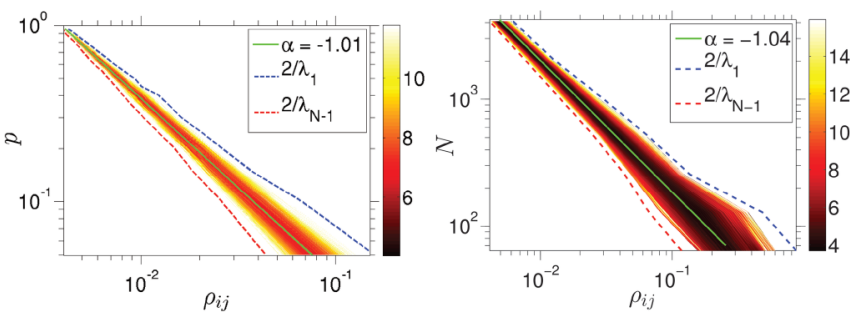

FIG. 2. (Color online) (a) [(b)] depicts how we modify random networks by adding edges [nodes]. Simulations initiate with a ring graph with $N=2^{9}$ nodes. Then, every pair of disjoint nodes is separately considered and linked with probability $p \in[0,1]$. The node addition is done by growing the ring graph (from $N=2^{6}$ to $2^{12}$ ) and then linking nodes as in the previous case with probability $p=10^{-1}$. The bottom panels show the resultant $\rho_{i j}$ PDF for both cases [edge addition in (c) and node addition in (d)] as it evolves when the control parameter ( $p$ or $N$, respectively) is increased as a function of the $\rho_{i j}$ values. The color scale corresponds to the logarithm of the $\rho_{i j}$ PDF. The analytical bounds derived in Eq. (4) are shown with dashed lines (see insets).

with low node degree (which lowers the magnitude of the spectral gap, hence increasing the upper bound for the edge capacity).

We particularize now the analysis of the effect of connectivity modifications (changes to $A_{i j}$ ) to the $\rho_{i j}$ probability distribution function (PDF) for two types of numerically generated networks: random (Fig. 2) [25] and small world (Fig. 3) [26]. In both cases, two growth protocols are carried out. The protocols and their effect on the $\rho_{i j}$ PDFs are shown in Figs. 2 and 3.

For random networks (RNs), the first protocol performs edge additions to a fixed ring network of $N=2^{9}$ nodes, namely, every pair of disjoint nodes in the ring is separately considered and linked with probability $p$ [Fig. 2(a)]. The second protocol for RNs grows the ring graph from $N=2^{6}$ to $2^{12}$ and, for each ring, disjoint nodes are linked as before with probability $p=10^{-1}$ [Fig. 2(b)]. Consequently, the first (a)

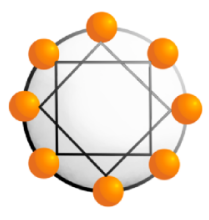

(b)
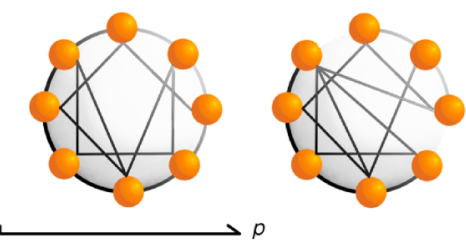

(c)
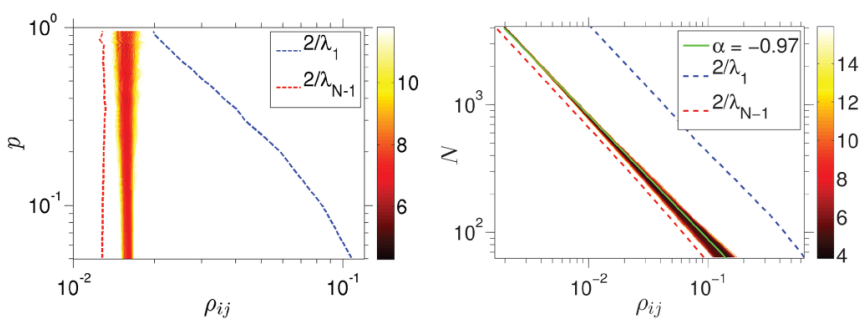

FIG. 3. (Color online) (a) depicts how we modify small-world networks by rewiring edges. Simulations initiate with a regular network of $N=2^{9}$ nodes with node degree $k=N / 4$. Then, each edge is rewired with probability $p \in[0,1]$. The node addition is done by growing the regular graph from $N=2^{6}$ to $2^{12}$ and then rewiring the edges with probability $p=10^{-1}$. The bottom panels show the resultant $\rho_{i j}$ PDF for both cases [edge rewiring in (b) and node addition in (c)] as it evolves when the control parameter is increased ( $p$ or $N$ ) as a function of the $\rho_{i j}$ values. The color scale and lines follow the same criteria as in Fig. 2.

protocol for RNs keeps the number of nodes fixed and increases the number of edges, hence increasing the edge density [the ratio between the existing number of edges to the total possible edges, i.e., $\simeq[p N(N-3)+2 N] / N(N-1) \stackrel{p}{\rightarrow} 1]$. On the other hand, the second protocol adds nodes and edges, but decreases the edge density $[\simeq[p N(N-3)+2 N] / N(N-$ $1) \stackrel{N}{\rightarrow} p$ ]. For small-world (SW) networks, the first protocol rewires the edges existing in a regular graph of $N=2^{9}$ nodes and node degree $k=N / 4$ with probability $p$ [Fig. 3(a)]. The second protocol increases the number of nodes in the regular graph from $N=2^{6}$ to $2^{12}$, and for each $N$, rewires the edges with probability $p=10^{-1}$. This means that for SW networks, the first protocol keeps the number of nodes, edges, and edge density $(2 k / N=1 / 2)$ fixed. On the other hand, the second protocol maintains the edge density fixed, but increases the number of nodes and edges.

Figures 2(c) and 2(d), and Figs. 3(b) and 3(c), show the probability distribution of $\rho_{i j}$ in logarithmic scale (color scale) as a function of the values at the edges $\left(\rho_{i j}\right)$ and the control parameter (either $p$ or $N$ ) for each topology evolution protocol. RNs and SW networks exhibit power-law behavior of the $\rho_{i j}$ PDFs for both growth protocols, with the exception of the edge rewiring protocol for a fixed number of nodes in SW networks [Fig. 3(b)]. In other words, most growth processes lead to a power-law distribution of the edge capacities as a function of the control parameter (either $p$ or $N$ ), as it also happens in scalefree networks [14]. Such topology modification protocols are the inverse of edge or node removals. In particular, if $Q$ nodes are added to a network of $N$ nodes following some protocol, it is the same as starting with a network of 
$N+Q$ nodes and removing $Q$ nodes following the same protocol. Thus, no different behavior in the $\rho_{i j}$ PDFs is expected.

This power-law behavior is very advantageous when designing an invariant flow distribution for an evolving supplydemand network as it allows one to predict the evolution of the capacities. Moreover, we find that at every step of the growth process, the edge capacity distribution is mainly given by the behavior of the most probable $\bar{\rho}$ value. Thus, its evolved magnitude can be predicted from the power-law exponents at any step. The evolution of $\bar{\rho}$ is derived from

$$
\bar{\rho}(r) \simeq e^{-\beta / \alpha} r^{1 / \alpha},
$$

where $\alpha<0$ and $\beta$ are the scaling exponents $[\log (r) \simeq$ $\alpha \log (\bar{\rho})+\beta]$ and the protocol control parameter $r$ is either $p$ or $N$. Furthermore, as can be seen from the insets of these figures, we find that $\alpha \sim-1$ for all of the power-law cases.

We interpret the power-law behavior in the following way. Any addition of edges to the topology results in a decrease of the equivalent resistance between nodes, hence a decrease in our theoretical edge capacity value. This phenomenon is a consequence of having more paths between nodes. In basic circuit theory, parallel paths are added as inverse summations, i.e., $1 / \rho_{i j}=1 / R_{i j}+\sum_{k>2} 1 / R_{i j}^{(k)}<1 / R_{i j}$, where $R_{i j}^{(k)}$ is the resistance of a path of length $k>2$ that joins nodes $i$ and $j$. This is why the edge and node addition protocols for RNs and the node addition protocol for SW networks result in a power-law PDF evolution and a decrease of the $\rho_{i j}$ values. When the connectivity modification protocol fixes the number of nodes, edges, and edge density [such as the protocol in Fig. 3(b)], then the $\rho_{i j}$ 's PDF remains invariant.

Any real supply-demand network operates by using manageable margins for their edge capacity values to avoid failures due to unpredictable fluctuations. Such fluctuations can be due to the switch from hub sources to distributed smaller producer [case (i)], the change in the location of suppliers and consumers [case (ii)], topology intended modifications [case (iii)], or directed attacks. Using Eq. (3), we provide a robust quantity that is not surpassed in either case (i) or case (ii).

In the cases where the knowledge of the full network structure is missing, we also obtain manageable bounds for the exact capacity values in terms of minimal information of the network structure, e.g., eigenvalues of the weighted Laplacian matrix [Eq. (4)] and minimum and maximum degrees (see Supplemental Material [18]). Our margins give simple engineering strategies for modifying the network's topology while bounding the capacities and maintaining a stable distribution.

To summarize, by analytically providing exact edge capacity values (plus bounds) of conservative linear flow problems, we are able to show how to design resilient supply-demand networks. In particular, we analyze the DC power-grid bounds for maximum load values and give a direct explanation of how to grow a numerically generated network by preserving its distribution stability, namely, by adding nodes and/or edges such that no cascade of failures occurs. In other words, we give exact [Eq. (3)] and approximate [Eq. (4)] protocols to evolve a network by maintaining the supply-demand flow stable.

The authors acknowledge the Scottish University Physics Alliance (SUPA).
[1] G. Kirchhoff, Ann. Phys. 148, 497 (1847).

[2] B. Bollobás, Modern Graph Theory (Springer-Verlag, New York, 1998).

[3] R. K. Ahuja, T. L. Magnanti, and J. B. Orlin, Network Flows: Theory, Algorithms, and Applications (Prentice-Hall, Englewood Cliffs, NJ, 1993), Chaps. 1 and 3.

[4] Y.-H. Chen, B.-H. Wang, L.-C. Zhao, C. Zhou, and T. Zhou, Phys. Rev. E 81, 066105 (2010).

[5] F. R. K. Chung, Spectral Graph Theory (Am. Math. Soc., CBMS 92, Providence, RI, 1997), Chaps. 1, 3, 4, 7.

[6] D. Randall, Comput. Sci. Eng. 6, 1521 (2006).

[7] M. E. J. Newman and M. Girvan, Phys. Rev. E 69, 026113 (2004).

[8] M. E. J. Newman, Eur. Phys. J. B 38, 321 (2004).

[9] N. Rubido, C. Grebogi, and M. S. Baptista, Europhys. Lett. 101, 68001 (2013).

[10] M. T. Brown, Ecol. Mod. 178, 83 (2004).

[11] E. Katifori, G. J. Szollosi, and M. O. Magnasco, Phys. Rev. Lett. 104, 048704 (2010).

[12] G. G. Batrouni and A. Hansen, Phys. Rev. Lett. 80, 325 (1998).

[13] Carlos Felipe Saraiva Pinheiro and A. T. Bernardes, Phys. Rev. E 72, 046709 (2005).
[14] E. López, S. V. Buldyrev, S. Havlin, and H. E. Stanley, Phys. Rev. Lett. 94, 248701 (2005).

[15] S. Carmi, Z. Wu, S. Havlin, and H. E. Stanley, Europhys. Lett. 84, 28005 (2008).

[16] R. Yang, W.-X. Wang, Y.-C. Lai, and G. Chen, Phys. Rev. E 79, $026112(2009)$.

[17] W.-X. Wang and Y.-C. Lai, Phys. Rev. E 80, 036109 (2009).

[18] See Supplemental Material at http://link.aps.org/supplemental/ 10.1103/PhysRevE.89.012801 for the Laplacian matrix eigenvalues and eigenvectors properties, the supply-demand network model and single source-sink solution, the edge capacity definition and proof, the many sources and sinks extension, and the edge and node capacity bounds.

[19] J. Cserti, Am. J. Phys. 68, 896 (2000).

[20] F. Y. Wu, J. Phys. A: Math. Gen. 37, 6653 (2004).

[21] A. Ghosh, S. Boyd, and A. Saberi, SIAM Rev. 50, 37 (2008).

[22] H. Chen and F. Zhang, Disc. App. Math. 155, 654 (2007).

[23] K. C. Das, A. D. Güngör, and A. S. Cevik, MATCH Commun. Math. Comput. Chem. 67, 541 (2012).

[24] www.nationalgrid.com/uk/Electricity/SYS/current.

[25] P. Erdös and A. Rényi, Publ. Math. 6, 290 (1959).

[26] D. J. Watts and S. H. Strogatz, Nature (London) 393, 440 (1998). 